\title{
Leptomeningeal metastasis of pulmonary large-cell neuroendocrine carcinoma: A case report and review of the literature
}

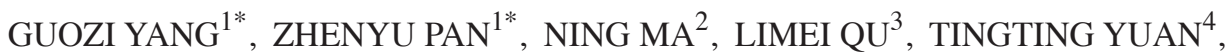 \\ XIAOCHUAN PANG ${ }^{5}, \mathrm{XU} \mathrm{YANG}^{1}$, LIHUA DONG $^{1}$ and SHIXIN LIU ${ }^{6}$ \\ Departments of ${ }^{1}$ Radiation-Oncology, ${ }^{2}$ Rheumatology, ${ }^{3}$ Pathology, ${ }^{4}$ Radiology and ${ }^{5}$ Clinical Laboratory, \\ The First Hospital of Jilin University, Changchun, Jilin 130021; ${ }^{6}$ Department of Radiation-Oncology, \\ Tumor Hospital of Jilin, Changchun, Jilin 130000, P.R. China
}

Received May 6, 2016; Accepted June 2, 2017

DOI: $10.3892 / 01.2017 .6676$

\begin{abstract}
Pulmonary large-cell neuroendocrine carcinoma (LCNEC) is a rare and malignant form of lung cancer with a poor prognosis for patients. The common sites of metastases are the liver, adrenal glands, bone and brain. LCNEC rarely metastasizes to the small intestine, ovaries, tonsils, mandible, vulva or spine. To the best of our knowledge, there have been no reports of leptomeningeal metastasis of LCNEC to date. The present case report describes an unusual case of leptomeningeal metastasis from pulmonary LCNEC alongside a review of the literature. Biopsies of pulmonary lesions and cervical lymph nodes confirmed the diagnosis of LCNEC in a 39-year-old male patient. At 2 months after chemotherapy, the patient began to experience hoarseness, epileptic seizures and blurred vision. Furthermore, the patient presented with radiating pain and numbness in his lower left limb. Imaging findings and cytological examination of cerebral spinal fluid supported the diagnosis of leptomeningeal metastasis. The patient's neurological symptoms were markedly alleviated following receipt of radiation and intrathecal chemotherapy. The patient survived for 4.9 months after diagnosis with leptomeningeal metastasis. To the best of our knowledge, the present case report is the first to describe leptomeningeal metastasis from pulmonary
\end{abstract}

Correspondence to: Dr Lihua Dong, Department of Radiation-Oncology, The First Hospital of Jilin University, 71 Xinmin Street, Changchun, Jilin 130021, P.R. China

E-mail: dlh@jlu.edu.cn

Dr Shixin Liu, Department of Radiation-Oncology, Tumor Hospital of Jilin, 1018 Huguang, Changchun, Jilin 130000, P.R. China

E-mail: liushixin1964@sina.com

${ }^{*}$ Contributed equally

Key words: leptomeningeal metastasis, large-cell neuroendocrine carcinoma, lung, magnetic resonance imaging, cytological examination
LCNEC confirmed by neuroimaging and cerebral spinal fluid cytology. It suggests that leptomeningeal metastasis does occur in this rare disease, and aggressive treatment may result in improved symptoms and possibly survival times.

\section{Introduction}

Pulmonary large-cell neuroendocrine carcinoma (LCNEC) is a rare type of lung cancer that accounts for $<1 \%$ of all primary lung malignancies (1). Since the biological behavior of LCNEC is similar to that of small cell lung cancer (SCLC), patients with LCNEC are often misdiagnosed and have an extremely poor prognosis (2). The 5-year survival rates for patients with LCNEC have been revealed to be significantly decreased compared with patients with other histological types of non-small cell lung cancer (NSCLC) $(2,3)$.

The common sites of metastasis from LCNEC are the liver, adrenal glands, bone, and brain, with hematogenous metastasis being the primary cause of treatment failure. Until recently, the rare sites of metastases from LCNEC which have been reported include the ovaries, small intestine, mandible, tonsil, vulva and prostate (4-9). In addition, Tsimpas et al (10) reported a case of LCNEC metastasis in the cauda equina; however, the patient in this study did not receive a cerebrospinal fluid (CSF) test and was not able to be diagnosed with leptomeningeal metastasis. Paydas et al (11) reported a case of leptomeningeal metastasis from LCNEC, but no cytological examination had been performed and no line-enhancement imaging for the diagnosis of leptomeningeal metastasis was performed for the patient. To the best of our knowledge, the present case report is the first to describe leptomeningeal metastasis from pulmonary LCNEC confirmed by neuroimaging and CSF cytology. Thus, the present case report describes a rare case of leptomeningeal metastasis from LCNEC alongside a review of the literature.

\section{Case report}

A 39-year-old male was admitted to Norman Bethune First Hospital (Changchun, China) in April 2013 complaining of a cough and blood-stained sputum that lasted for 10 days. 


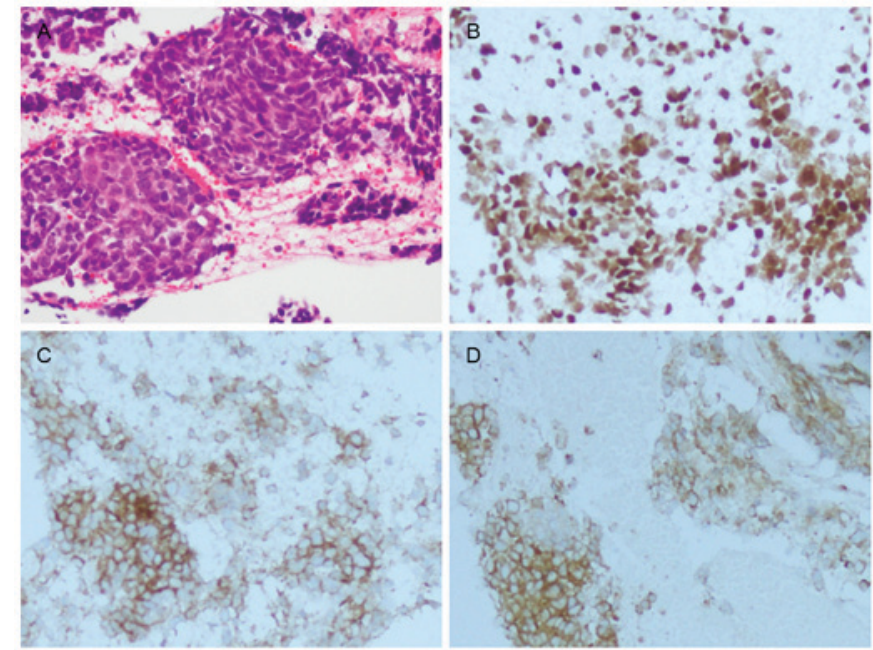

Figure 1. Pathological examination of pulmonary large-cell neuroendocrine carcinoma tissue. Lung tumors were visualized using a bronchoscope and biopsied. (A) Hematoxylin and eosin staining revealed unclear boundaries with infiltrative growth and rich in cells which were arranged as flaky, cord-like, adenoid or chrysanthemum-shaped clusters. The cells commonly presented with large volumes with a polygonal shape, small cytoplasm, filamentous chromatin and intensely stained nuclei. Three to four abnormal mitotic events may be observed in each high-power field. A range of tumor necrosis may be visualized in the area of poor differentiation. Pulmonary large-cell neuroendocrine tumor cells were positive for the neuroendocrine-specific markers (B) thyroid transcription factor-1, (C) cluster of differentiation 56 and (D) synaptophysin. Magnification, x400.

The patient had a 20-year history of drinking and smoking. A computed tomography (CT) scan of the chest revealed a mass in the right pulmonary hilum that was accompanied by an enlargement of the right supraclavicular and mediastinal lymph nodes. A transbronchial biopsy revealed that the tumor cells exhibited an unclear boundary, with infiltrative growth and numerous cells which were arranged as flaky, cord-like, adenoid or chrysanthemum-shaped clusters. The cells commonly presented with large volumes, polygonal shape, small cytoplasm, filamentous chromatin and intensely stained nuclei. Three to four abnormal mitotic events were observed in each high-power field. A wide range of tumor necrosis was visualized in the area of poor differentiation (Fig. 1A). Staining of the patient's tumor was positive for thyroid transcription factor-1 (TTF-1), cluster of differentiation 56 (CD56; also known as neural cell adhesion molecule), synaptophysin (Syn) (Fig. 1B-D) and p63 (interspersed), and negative for leucocyte common antigen. This supported the histological diagnosis of LCNEC. Pathological examination of the right supraclavicular lymph node indicated metastatic cancer invasion (Fig. 2A). This observation was confirmed by negative immunohistochemical staining for cytokeratin $5 / 6$, and positive staining for TTF-1, CD56, Syn (Fig. 2B-D) and p63 (partial). This supported the histological diagnosis of a neuroendocrine carcinoma with a pulmonary origin. No other metastases were detected using an abdominal CT scan.

The patient had a partial response to six cycles of systemic chemotherapy with gemcitabine (2 g on day 1$)$ and cisplatin (50 mg on days 1-3). The lesion decreased in size following two cycles of chemotherapy and the patient achieved partial disease control. No severe adverse effects were detected. At 2 months after the last chemotherapy cycle, the patient

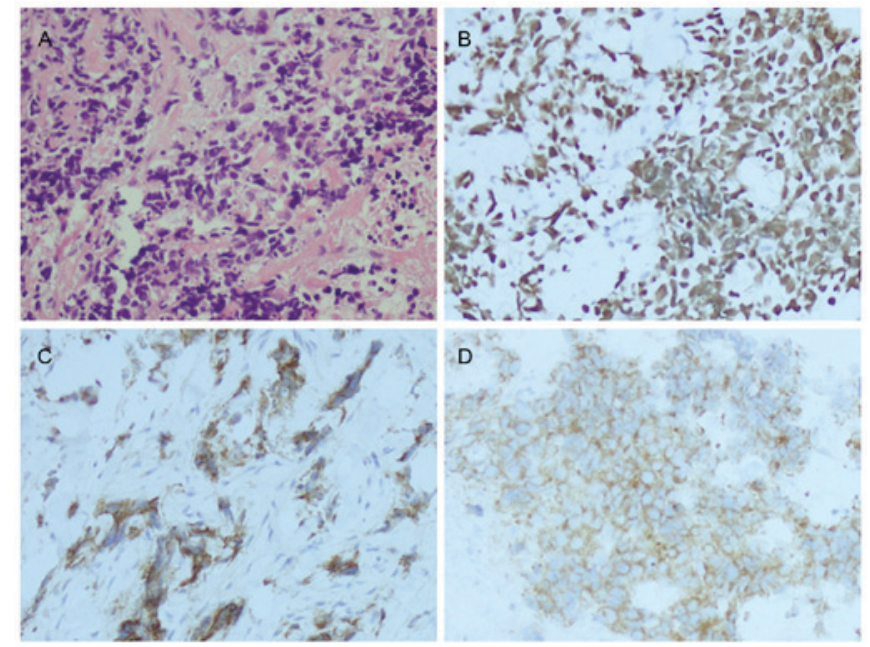

Figure 2. Pathological examination of the supraclavicular lymph nodes. Pulmonary LCNEC cells that migrated to the lymph nodes were biopsied. (A) Hematoxylin and eosin staining revealed that tumor cells exhibited a large cellular volume, markedly stained nuclei and a moderate amount of cytoplasm. LCNEC cells were positive for the neuroendocrine markers (B) thyroid transcription factor-1, (C) cluster of differentiation 56 and (D) synaptophysin. Magnification, x400. LCNEC, large-cell neuroendocrine carcinoma.

began to experience radiating pain and numbness in his left lower limb, hoarseness, epileptic seizures and blurred vision. Additionally, the patient suffered a seizure due to symptom aggravation. Conventional biochemical examinations including routine blood, urine, liver and kidney function tests were normal. Serum levels of tumor markers were $22.31 \mathrm{U} / \mathrm{ml}$ cancer antigen 153 (CA153), $1.16 \mathrm{ng} / \mathrm{ml}$ carcinoembryonic antigen (CEA), $14.6 \mathrm{ng} / \mathrm{ml}$ neuron-specific enolase (NSE) and $0.7 \mathrm{ng} / \mathrm{ml}$ CYFRA21-1. A CT scan of the brain revealed irregular low-density shadows from edema on the right parietal-occipital area (Fig. 3A). Magnetic resonance imaging (MRI) of the brain identified metastatic nodules in the inferior cortex, sulci and gyri and internal ventricles, and line-enhancements in the leptomeningeal mater (Fig. 3B-D). Gadolinium enhancement scans of the lumbar spine revealed flake-enhanced lesions in the T12 vertebra of the spine and nodular-enhanced lesions along the cauda equina nerve in the L3-4 space (Fig. 3E). A low signal nodule-like lesion along the cauda equina nerve was observed using T2-weighted imaging (T2WI; Fig. 3F). Results from a lumbar puncture revealed that the patient's CSF was colorless, intracranial pressure was $200 \mathrm{mmH}_{2} \mathrm{O}$, the protein level was $0.75 \mathrm{~g} / \mathrm{l}$ and the glucose level was $3.35 \mathrm{mmol} / \mathrm{l}$. Tumor cells were identified within the patient's CSF via liquid-based technology (ThinPrep TCT2000) combined with Papanicolaou staining (Fig. 4). Tumor marker levels in CSF were $1.00 \mathrm{U} / \mathrm{ml} \mathrm{CA153,0.20} \mathrm{ng/ml}$ CEA, $25.8 \mathrm{ng} / \mathrm{ml} \mathrm{NSE}$ and $2.5 \mathrm{ng} / \mathrm{ml}$ CYFRA21-1. On the basis of these results, a diagnosis of leptomeningeal metastasis was made. The patient's Karnofsky performance status (KPS) (12) score was determined to be $40-50$ points.

The patient's treatment regimen involved three-dimensional conformal radiotherapy with a $6 \mathrm{MV}$ X-ray to the whole brain and thoracolumbar spinal canal, which consisted of a 40 Gy total radiation dose administered in 20 fractions over a 4 -week period. Simultaneous administration of 

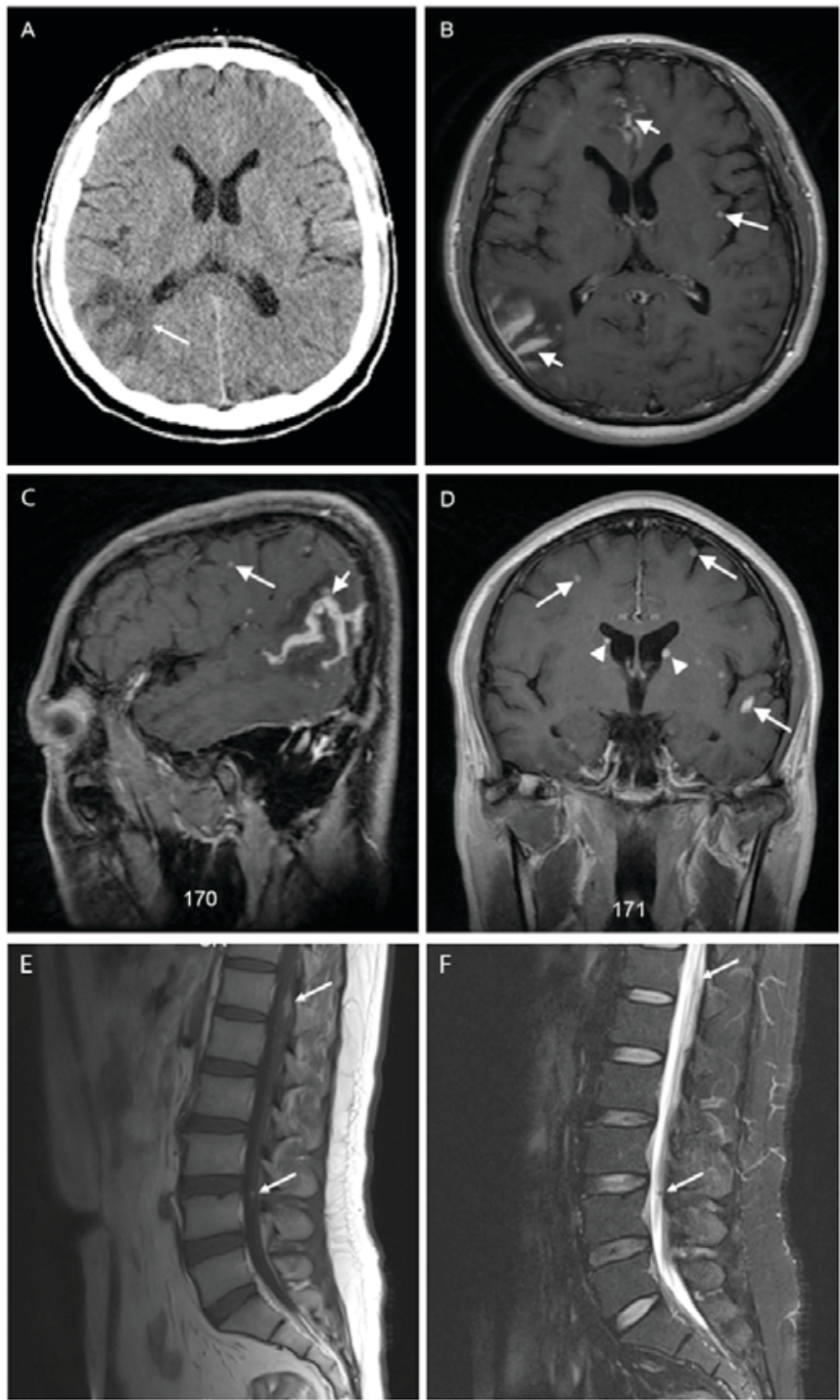

Figure 3. Brain and lumbar spine images. (A) CT scan of the brain revealed irregular low-density shadows from edema on the right parietal-occipital area. (B) Axial MRI of the brain, including line-enhancements in the leptomeningeal mater (short arrows) and metastatic nodules in the sulci and gyri (long arrow). (C) Sagittal MRI of the brain: line-enhancements in the leptomeningeal mater (short arrow) and metastatic nodules in the sulci and gyri (long arrow). (D) Coronal MRI of the brain: metastatic nodules in the sulci and gyri (long arrows) and metastatic nodules in internal ventricles (arrowheads); (E) Gadolinium enhancement scan of the lumbar spine revealed flake-enhanced lesions in the T12 vertebrae of the spine, nodular-enhanced lesions along the cauda equina nerve at the L3-4 level. (F) A low signal nodule-like lesion along the cauda equina nerve was observed using T2-weighted imaging. CT, computed tomography; MRI, magnetic resonance imaging.

intrathecal chemotherapy using methotrexate (MTX, $15 \mathrm{mg}$ ) and dexamethasone $(5 \mathrm{mg})$ was performed once a week. The first intrathecal chemotherapy began on the first day of radiotherapy. Three cycles of intrathecal chemotherapy and 14 days of radiotherapy ( $20 \mathrm{~Gy}$ in 10 fractions) markedly alleviated the patient's symptoms and his KPS score increased to 60 points. The patient declined further treatment for personal reasons and was discharged from the hospital. After 2 months, the patient began to experience headaches and numbness and succumbed due to disease progression. The patient's overall survival (OS) time was 11 months, and the patient had survived

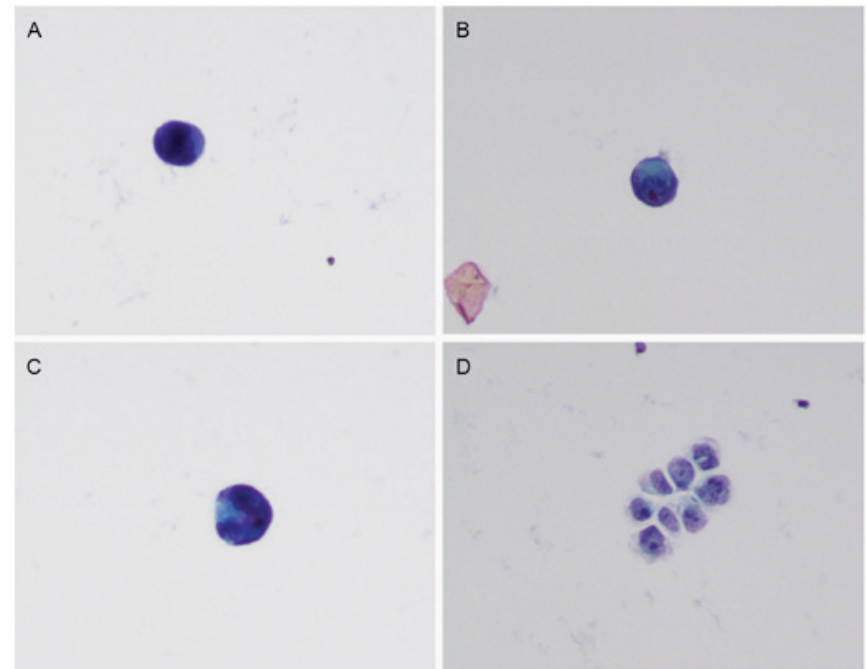

Figure 4. Cytological analysis of CSF. Tumor cells were predominantly scattered (A-C), and commonly exhibited (A) large single nuclei, (B) an irregular shape and (C) a large volume with deep staining. Rarely, (C) multiple nuclei and (D) clusters were also observed. Magnification, x400. CSF, cerebrospinal fluid.

for 4.9 months from the time of diagnosis of leptomeningeal metastasis.

Written informed consent was obtained from the next of kin of the patient for publication of this case report and any accompanying images.

\section{Discussion}

Leptomeningeal metastasis is a fatal complication of malignant cancers and occurs in 5\% of patients diagnosed with solid tumors (13). This type of metastasis results from invasion of the subarachnoid space by the migratory tumor cells and dissemination through the CSF. Thus, patients usually have pleomorphic and multifocal neurological complaints due to disperse involvement of the central nervous system. Despite having an aggressive treatment regimen, the median OS time is between 2 and 3 months (13). Leptomeningeal metastasis often occurs in patients with melanoma, breast or lung cancer. Although lung cancer is one of the most common malignant solid tumors prone to invade the meninges, to the best of our knowledge, there has been no report concerning leptomeningeal metastasis from pulmonary LCNEC. To the best of our knowledge, the present case report documents the first cytologically confirmed case of leptomeningeal involvement from LCNEC.

In 1991, Travis et al (14) first described the histological characteristics of LCNEC, which included large cells with abundant cytoplasm, a high mitotic rate, extensive necrosis and a neuroendocrine growth pattern. In 2001, the World Health Organization suggested that, in order to confirm the neuroendocrine origin of the tumor cells and thereby diagnose LCNEC, a neuroendocrine morphology and positive immunohistochemical staining for at least one neuroendocrine-specific marker, e.g. chromogranin, CD56 or Syn, must be present (15). In the present case report, histopathological examination of the patient's primary lung tumor and cervical lymph nodes 
combined with immunohistochemistry confirmed the diagnosis of LCNEC.

An MRI examination is a critical auxiliary diagnosis for leptomeningeal metastasis (13). The major imaging features include dot- and line-enhancements in the leptomeningeal mater, metastatic nodules in the sulci and gyri, inferior cortex, internal ventricles and seeding nodules along the cauda equina nerve (13). In the present case report, the patient exhibited all of the clinical imaging manifestations mentioned above that conformed to the characteristics of implantation metastases. In particular, line-enhancements in the sulci and gyri and implanted metastatic nodules along the cauda equina nerve are considered specific imaging features for leptomeningeal metastasis, which may be used as a diagnostic tool (14).

CSF cytological analysis provides the optimum assessment of leptomeningeal metastasis (13). In this case, the tumor cells exhibited evident characteristics of malignancy that included large cellular volumes, pleomorphism, markedly increased nuclear-to-cytoplasmic ratio and markedly stained chromatin.

There are a limited number of reports describing the treatment regimens for patients with LCNEC. Several studies have demonstrated that the response rate of LCNEC to cisplatin-based chemotherapy was similar to that of small cell carcinoma (16). In 2013, a multicenter prospective study reported the median progression-free survival and OS time for 42 patients with advanced stage LCNEC were 5.2 months and 7.7 months respectively, following cisplatin-irinotecan chemotherapy (17).

Patients with leptomeningeal metastasis, which is a fatal complication of malignant tumor, have a very poor prognosis. The main objective of leptomeningeal metastasis treatment is to alleviate symptoms of the nervous system, improve quality of life and prolong the survival time of the patient (13). Owing to the direct exposure of the central nervous system to cancer cells, a whole central nervous system therapy using intrathecal chemotherapy alone or in combination with local radiotherapy should be performed (13). MTX remains the most widely used and clinically effective intrathecal chemotherapeutic drug used to treat leptomeningeal metastasis from solid tumors (13). Currently, an intrathecal injection of 10-15 mg MTX twice weekly is more commonly used in the initial treatment (13). Radiotherapy on bulky disease observed on MRI or sites of symptomatic disease eliminates locally aggregated tumor cells and re-establishes the normal CSF circulation to improve the efficacy, as well as decrease the toxicity of intrathecal chemotherapy. In addition, metastatic lesions in the brain parenchyma may also be effectively treated simultaneously (13). The regimen of whole brain radiotherapy commonly consists of a total radiation dose of $30 \mathrm{~Gy}$ in 10 fractions for 2 weeks (13). In the present case, the patient received simultaneous intrathecal chemotherapy with radiotherapy. To reduce neurotoxicity, the single radiotherapy dosage was set at 2 Gy and the density of the regimen of intrathecal MTX was reduced to once per week. The treatment was well tolerated by the patient and the symptoms were alleviated rapidly. There was no severe adverse reaction. However, the patient failed to complete all treatments owing to personal reasons and succumbed to disease progression.

Currently, there is no standard therapy regimen for leptomeningeal metastasis from solid tumors. Intrathecal chemotherapy and radiotherapy are valuable treatment approaches, but an optimal combination of distinct treatments has not been extensively studied. The male patient in the present case report presented multiple adverse prognostic factors including a low KPS score as well as severe and pleomorphism nerve dysfunction. Previous studies indicated that intrathecal chemotherapy does not improve OS times in solid tumors (18-20) and National Comprehensive Cancer Network (NCCN) guidelines suggest that radiation therapy alone may produce a positive effect with less toxicity. However, studies have demonstrated that radiation therapy alone only alleviated symptoms of the nervous system and did not prolong patient OS times $(21,22)$.

NCCN guidelines suggest the use of simultaneous intrathecal chemotherapy and radiation in leptomeningeal metastasis, but this combination treatment has not been extensively studied. Therefore, this approach was adopted to treat leptomeningeal metastasis in patients with adverse prognostic factors. Untreated patients with leptomeningeal metastasis have a median survival time of 4-6 weeks; this survival time may be prolonged to 2-3 months in patients with NSCLC from leptomeningeal metastasis by using effective treatments (13). In the present case report, the patient did not suffer obvious toxic effects and survived for 4.9 months from the time of diagnosis of leptomeningeal metastasis. This time was longer than the median survival time previously reported, suggesting a benefit of administration of simultaneous treatments to alleviate neurological symptoms and extend survival times.

In conclusion, pulmonary LCNEC with leptomeningeal metastasis is a rare disease that is associated with poor prognosis. Nevertheless, the present case report and review of the literature suggest that doctors should realize the potential of leptomeningeal metastasis from pulmonary LCNEC, and aggressive treatment may result in improved symptoms and possibly survival.

\section{Acknowledgements}

The authors wish to thank Dr Yongxiang Wang for her expert technical assistance with cytological analysis of cerebrospinal fluid.

\section{References}

1. Gollard R, Jhatakia S, Elliott M and Kosty M: Large cell/neuroendocrine carcinoma. Lung Cancer 69: 13-18, 2010.

2. Sun JM, Ahn MJ, Ahn JS, Um SW, Kim H, Kim HK, Choi YS, Han J, Kim J, Kwon OJ, et al: Chemotherapy for pulmonary large cell neuroendocrine carcinoma: Similar to that for small cell lung cancer or non-small cell lung cancer? Lung Cancer 77: 365-370, 2012

3. Le Treut J, Sault MC, Lena H, Souquet PJ, Vergnenegre A Le Caer H, Berard H, Boffa S, Monnet I, Damotte D and Chouaid C: Multicentre phase II study of cisplatin-etoposide chemotherapy for advanced large-cell neuroendocrine lung carcinoma: The GFPC 0302 study. Ann Oncol 24: 1548-1552, 2013.

4. Bugiantella W, Cavazzoni E, Graziosi L, Valiani S, Franceschini MS and Donini A: Small bowel metastasis from lung cancer: A possible cause of acute abdomen. Case report and literature review. G Chir 32: 120-122, 2011.

5. Murakawa T, Nakajima J, Fukami T, Tanaka M, Takeuchi E and Takamoto S: Tonsillar metastasis from large cell carcinoma of the lung. Jpn J Thorac Cardiovasc Surg 49: 377-380, 2001. 
6. Nelson BE, Carcangiu ML and Chambers JT: Intraabdominal hemorrhage with pulmonary large cell carcinoma metastatic to the ovary. Gynecol Oncol 47: 377-381, 1992.

7. Rocconi RP, Leath CA III, Johnson WM III, Barnes MN III, Conner MG: Primary lung large cell carcinoma metastatic to the vulva: A case report and review of the literature. Gynecol Oncol 94: 829-831, 2004.

8. Shimizu K, Goto T, Maeshima A, Oyamada Y and Kato R: Prostatic metastasis of pulmonary large cell neuroendocrine carcinoma. J Cancer 3: 96-99, 2012.

9. Yoshii T, Muraoka S, Sano N, Furudoi S and Komori T: Large cell carcinoma of the lung metastatic to the mandibular gingiva. J Periodontol 73: 571-574, 2002.

10. Tsimpas A, Post NH, Moshel Y and Frempong-Boadu AK: Large cell neuroendocrine carcinoma of the lung metastatic to the cauda equina. Spine J 10: e1-e5, 2010.

11. Paydas S, Bicakci K and Yavuz S: Dramatic response with capecitabine after cranial radiation to the brain parenchymal and leptomeningeal metastases from lung cancer. Eur J Intern Med 20: 96-99, 2009.

12. Schag CC, Heinrich RL and Ganz PA: Karnofsky performance status revisited: Reliability, validity, and guidelines. J Clin Oncol 2: 187-193, 1984

13. Le Rhun E, Taillibert S and Chamberlain MC: Carcinomatous meningitis: Leptomeningeal metastases in solid tumors. Surg Neurol Int 4 (Suppl 4): S265-S288, 2013.

14. Travis WD, Linnoila RI, Tsokos MG, Hitchcock CL, Cutler GB Jr, Nieman L, Chrousos G, Pass H and Doppman J: Neuroendocrine tumors of the lung with proposed criteria for large-cell neuroendocrine carcinoma. An ultrastructural, immunohistochemical, and flow cytometric study of 35 cases. Am J Surg Pathol 15: $529-553,1991$.
15. Brambilla E, Travis WD, Colby TV, Corrin B and Shimosato Y: The new World Health Organization classification of lung tumours. Eur Respir J 18: 1059-1068, 2001.

16. Yamazaki S, Sekine I, Matsuno Y, Takei H, Yamamoto N, Kunitoh H, Ohe Y, Tamura T, Kodama T, Asamura H, et al: Clinical responses of large cell neuroendocrine carcinoma of the lung to cisplatin-based chemotherapy. Lung Cancer 49: 217-223, 2005.

17. Niho S, Kenmotsu H, Sekine I, Ishii G, Ishikawa Y, Noguchi M, Oshita F, Watanabe S, Nakajima R, Tada $\mathrm{H}$ and Nagai $\mathrm{K}$ : Combination chemotherapy with irinotecan and cisplatin for large-cell neuroendocrine carcinoma of the lung: A multicenter phase II study. J Thorac Oncol 8: 980-984, 2013.

18. Chamberlain MC, Glantz M, Groves MD and Wilson WH: Diagnostic tools for neoplastic meningitis: Detecting disease, identifying patient risk, and determining benefit of treatment. Semin Oncol 36 (4 Suppl 2): S35-S45, 2009.

19. Chamberlain MC, Tsao-Wei D and Groshen S: Neoplastic meningitis-related encephalopathy: Prognostic significance. Neurology 63: 2159-2161, 2004.

20. Brem SS, Bierman PJ, Black P, Blumenthal DT, Brem H, Chamberlain MC, Chiocca EA, DeAngelis LM, Fenstermaker RA, Fine HA, et al: Central nervous system cancers: Clinical practice guidelines in oncology. J Natl Compr Canc Netw 3: 644-690, 2005.

21. Gani C, Müller AC, Eckert F, Schroeder C, Bender B, Pantazis G, Bamberg $\mathrm{M}$ and Berger B: Outcome after whole brain radiotherapy alone in intracranial leptomeningeal carcinomatosis from solid tumors. Strahlenther Onkol 188: 148-153, 2012.

22. Morris PG, Reiner AS, Szenberg OR, Clarke JL, Panageas KS Perez HR, Kris MG, Chan TA, DeAngelis LM and Omuro AM: Leptomeningeal metastasis from non-small cell lung cancer: Survival and the impact of whole brain radiotherapy. J Thorac Oncol 7: 382-385, 2012. 